Received: 2016.12.12 Accepted: 2017.03.24 Published: 2017.06.26

\title{
Extraovarian Primary Peritoneal Carcinomatosis: A Case Report
}

Authors' Contribution: Study Design A Data Collection B Statistical Analysis C Data Interpretation D Manuscript Preparation E Literature Search F Funds Collection G
ACDEF 1 Gregory Nicolas

AEFG 2 Tony Kfoury

BDFG 2 Hassan Fawaz

DFG 2 Mahdi Issa
1 Medical Student, LAUMRCH, Beirut, Lebanon

2 Department of Surgery, Mount Lebanon Hospital, Beirut, Lebanon
Corresponding Author: Conflict of interest:
Gregory Nicolas, e-mail: gregory.nicolas@lau.edu

None declared

Patient: Female, 66

Final Diagnosis: Extraovarian primary peritoneal carcinomatosis

Symptoms: Abdominal distension with constipation of 3 days, while still passing flatus. She further complained of mild postprandial epigastric pain, but denied nausea or vomiting as well as fever

\section{Clinical Procedure: $\quad-$ \\ Specialty: Surgery}

\section{Objective: Rare disease}

Background: Extraovarian primary peritoneal carcinoma (EOPPC) is a rare malignant epithelial tumor with an age-adjusted incidence rate of 6.78 per million, which arises from the peritoneal lining with minimal or no ovarian involvement. EOPPC is a diagnosis of exclusion with the absence of other identifiable primary sites and after surgical assessment and consideration of the histological report to evaluate the extent of ovarian invasion.

Case Report: $\quad$ A 66-year-old woman complained of mild postprandial epigastric pain. Physical exam revealed positive bowel sounds over all 4 quadrants, with a soft, non-tender abdomen. Distension and dullness to percussion were noted. A CT scan revealed peritoneal fluid, and evaluation of the peritoneal fluid showed an elevated white blood cell count, while fulfilling criteria for exudate. There was no bacterial growth from the peritoneal fluid. Serum tumor markers CEA were significantly elevated, suggestive of an underlying malignancy. An exploratory laparoscopy confirmed diffuse carcinomatosis in the omentum, the coloparietal region bilaterally, as well as surrounding small bowel loops, the transverse and sigmoid colon, and the rectum. A bilateral oophorectomy was performed and 2 biopsies from the thickened peritoneum were taken. The histomorphological features from the pathology examination concluded the patient had peritoneal serous carcinoma with uninvolved ovaries.

Conclusions: $\quad$ EOPPC may be misdiagnosed as epithelial ovarian cancer, and should be considered in patients with peritoneal carcinomatosis, normal-sized ovaries, and no identifiable primary lesion. Since the common therapeutic strategies achieve comparable survival rates, failed recognition may be without consequences. However, to further improve survival, optimal cytoreductive surgery is essential and should be made a priority in disease management.

MeSH Keywords: $\quad$ Laparoscopes • Medical Oncology・ Oncology Service, Hospital

Full-text PDF: http://www.amjcaserep.com/abstract/index/idArt/902801

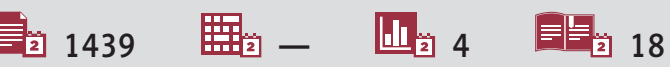




\section{Background}

Extraovarian primary peritoneal carcinoma (EOPPC) is a rare malignant epithelial tumor with an age-adjusted incidence rate of 6.78 per million [1], which arises from the peritoneal lining with minimal or no ovarian involvement.

In 1959, Swerdlow reported a peritoneal mass in a 27 -year-old woman with uninvolved ovaries and fallopian tubes, histologically similar to papillary serous carcinoma of the ovary, and named it "mesothelioma of the pelvic peritoneum" [2]. In 1977, Kannerstein et al. called for distinguishing the disease from malignant mesothelioma, which does not share the epidemiologic and biologic feature of primary peritoneal carcinoma [3].

EOPPC is a diagnosis of exclusion with the absence of other identifiable primary sites and after surgical assessment and consideration of the histological report to evaluate the extent of ovarian invasion.

In 1993, the Gynecologic Oncology Group defined criteria to distinguish EOPPC from serous adenocarcinoma of the ovary:

I. Both ovaries must be either normal in size or enlarged by a benign process;

II. The involvement in extraovarian sites must be greater than the involvement on the surface of either ovary;

III. Microscopically, the ovarian component must be either nonexistent, have no signs of cortical invasion, or have a tumor size less than $5 \mathrm{~mm}^{2}$ within ovarian substance with or without surface disease;

IV. The histological and cytological characteristics of the tumor must be predominantly of the serous type that is similar to ovarian serous cancer of any grade [4].

However, EOPPC does not have a separate staging system, making the application of the FIGO staging system necessary. Due to the peritoneal involvement, all patients are at least considered stage III or IV. Metastasis usually occurs transperitoneally, but lymphatic spread is frequent $(63-73 \%)[5,6]$.

The peritoneum shares a common embryonal heritage with ovarian tissue, deriving from coelomic epithelium just as the Müllerian ductal system does [7]. The retained potential for Müllerian differentiation may explain the development of primary malignant entities similar to epithelial ovarian cancer along the peritoneal lining. Despite the biological proximity of the tissues, the final answer to the pathological origin of the disease remains to be found.

Differentiation to peritoneal carcinomatosis from metastatic gastrointestinal cancers and advanced-stage epithelial ovarian cancer is important for planning the surgical approach.
Differential diagnosis includes metastatic peritoneal carcinomatosis, malignant mesothelioma, peritoneal psammocarcinoma, and endosalpingiosis, as well as other benign or borderline diseases. Malignant mesothelioma is linked to a history of asbestos exposure and occurs more frequently in males. [8] Distinction from primary peritoneal carcinoma can be supported by immunohistochemistry with malignant mesothelioma staining positive for $\mathrm{h}-\mathrm{CD}$ an Calretinin and virtually never for mucin [9]. Estrogen receptor (ER) positivity was found to be positive in $86 \%$ of women with EOPPC and $88 \%$ of women with epithelial ovarian cancer with no ER-expression in malignant mesothelioma.

Survival in EOPPC patients remains poor, with Eltabbakh et al. reporting a median overall survival of 23.5 months [10], and 5 year-survival-rates in the literature ranging from $0 \%$ to $26.5 \%$ [9].

\section{Case Report}

A 66-year-old woman, known to have dyslipidemia and osteoporosis, presented for abdominal distension with constipation of 3 days, while still passing flatus. She further complained of mild postprandial epigastric pain, but denied nausea, vomiting, and fever. Concerning her past surgical history, the patient had an open cholecystectomy 20 years prior to presentation.

The physical exam revealed positive bowel sounds over all 4 quadrants, with a soft, non-tender abdomen. Distension and dullness to percussion were noted.

The laboratory results on admission were inconspicuous, and a CT scan of the abdomen was scheduled to further investigate the complaints (Figures 1, 2), which revealed slight thickening of small bowel loops with no visible air fluid level. Moderate amounts of peritoneal fluid were found in the perihepatic and perisplenic space as well as in the pouch of Douglas. There was infiltration of the mesenteric fat, with diffuse enlargement of the omentum.

The patient was admitted under antibiotic chemotherapy and scheduled for ultrasound-guided ascites puncture. Evaluation of the peritoneal fluid showed an elevated white blood cell count of 1855 cells/ $\mu \mathrm{l}(<500$ cells $/ \mu$ l) while fulfilling criteria for exudate with a protein content of $53 \mathrm{~g} / \mathrm{L}(<30 \mathrm{~g} / \mathrm{L})$. There was no bacterial growth from peritoneal fluid or blood cultures; PCR for tuberculosis was negative. Serum tumor markers CEA of $11.3 \mu \mathrm{g} / \mathrm{L}$ (non-smoker, $0-3 \mu \mathrm{g} / \mathrm{L}$ ) and CA-125 of $1071 \mu \mathrm{g} / \mathrm{L}$ (0-35 $\mu \mathrm{g} / \mathrm{L})$ were significantly elevated, suggestive of an underlying malignancy. 


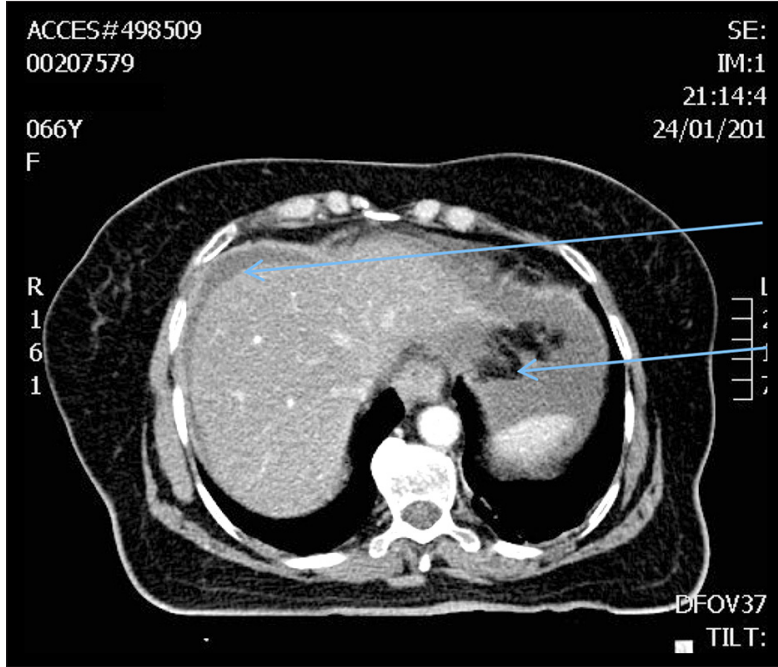

Figure 1. Abdominal CT scan, perihepatic and perisplenic fluid collection.

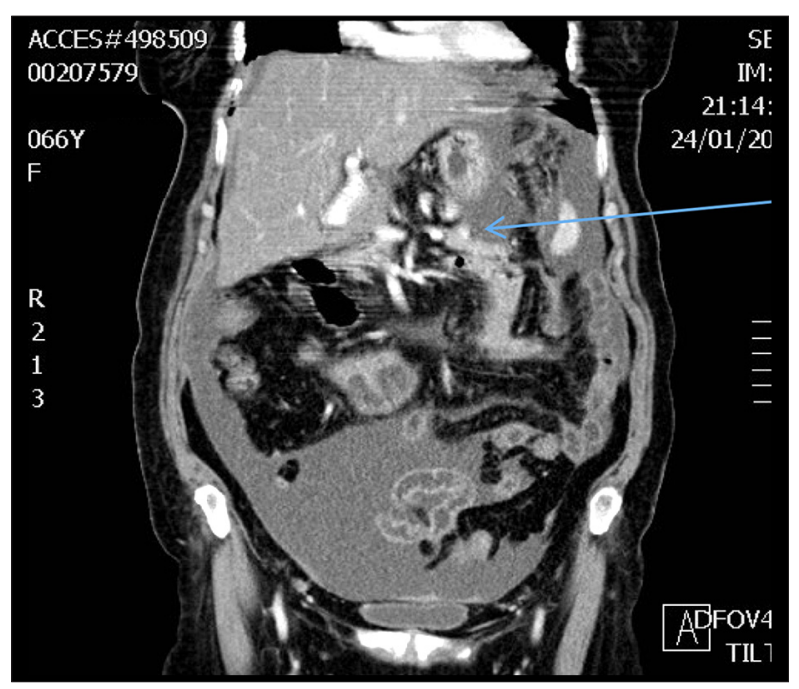

Figure 2. Abdominal CT scan, pelvic fluid collection.

A subsequent PET/CT scan with application of ${ }^{18} \mathrm{~F}-\mathrm{FDG}$ evoked the image of an intensely tracer-avid peritoneal carcinomatosis without signs of another identifiable primary disease (Figure 3).

An exploratory laparoscopy confirmed diffuse carcinomatosis in the omentum and the coloparietal region bilaterally, as well as surrounding small bowel loops, the transverse and sigmoid colon, and the rectum. A bilateral oophorectomy was performed and 2 biopsies from the thickened peritoneum were taken. The histomorphological features from the pathology examination concluded peritoneal serous carcinoma with uninvolved ovaries (Figure 4).

The patient completed 6 cycles of systemic chemotherapy using Carboplatin combined with Paclitaxel and Bevacizumab. $A$ repeat $\mathrm{CT}$ scan showed carcinomatosis, and a decrease in

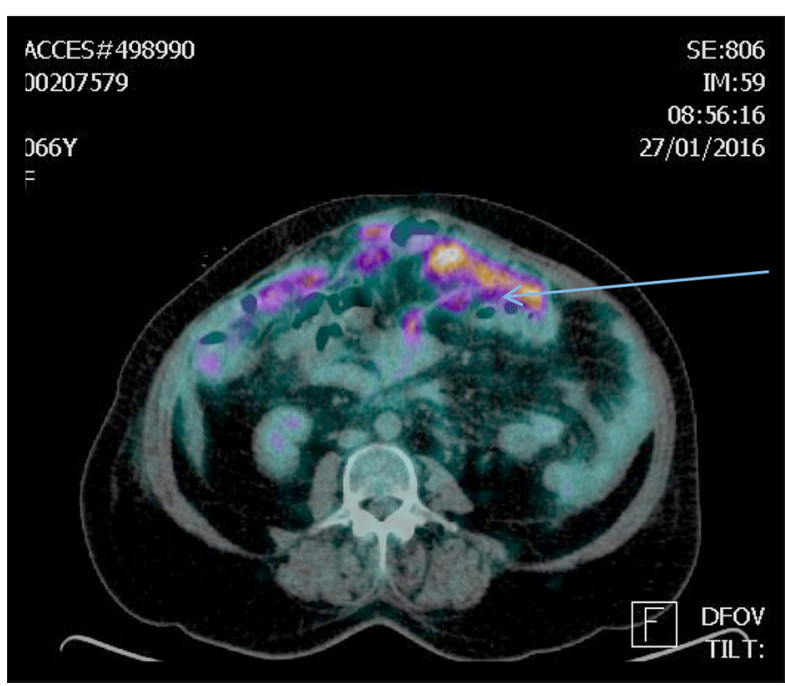

Figure 3. PET-CT scan.

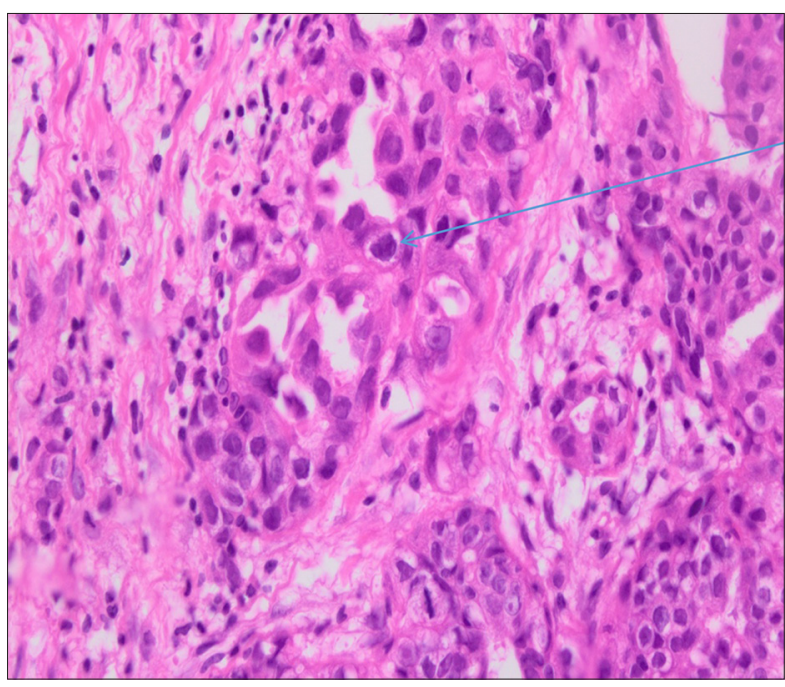

Figure 4. Hematoxylin and eosin staining of the omental specimen.

CA-125 to $52 \mu / L$ was noted. The patient was scheduled for debulking surgery after radiography, which is a marker of clinical response.

Exploration of the abdominal cavity revealed completely resectable disease, with flat disease $(<0.5 \mathrm{~mm})$ in $\mathrm{PCl}$ regions 0 , $1,2,6$, and $12(\mathrm{PCI} 6)$. The patient received a radical hysterectomy, followed by resection of the omentum and the appendix. A peritonectomy over the right Gerota's fascia and stripping of the right hemi-diaphragm in toto were performed. Furthermore, mesocolic and small bowel nodules were treated by meticulous resection and ablation, respectively. No complications arose during the procedure, which resulted in no residual visual disease. 
The postoperative course was uneventful and the patient was discharged on the $5^{\text {th }}$ postoperative day.

The pathology report confirmed the diagnosis of well-differentiated peritoneal papillary serous carcinoma (G1, pT3A). Lymphatic vascular invasion was noted, with few intralymphatic tumor emboli.

The patient presented 2 weeks after the debulking surgery with back and abdominal pain, and a CT scan revealed bibasilar atelectatic changes of the lung parenchyma, with a thick atelectatic band on the left. Symptoms were relieved by analgetic treatment and the patient was discharged shortly thereafter.

Two months after debulking surgery, no signs of relapse were noted.

After 1-year follow-up, abdomen and pelvis CT scans were normal, with no carcinomatosis or symptoms. We used CT scanning for the diagnosis, which is more sensitive and specific than ultrasound.

\section{Discussion}

Since EOPPC has to be distinguished from differential malignant diseases, surgical findings and histopathological examination are obligatory for diagnosis. The clinical presentation may consist of nonspecific symptoms like abdominal pain, distension, and constipation, as in our patient. Other symptoms may include gastrointestinal symptoms (e.g., nausea/vomiting and dyspepsia), change in weight, and abdominal or pelvic masses, overlapping the symptomatic spectrum of ovarian cancer and thus rendering it indistinguishable without further workup.

Radiological imaging is a valuable tool for guiding surgical management. Even without final differentiation between malignancies, the use of PET/CT employing ${ }^{18} \mathrm{~F}-\mathrm{FDG}$ has been well established for assessing the biological activity and presence of a suspicious mass [11].
Remarkable elevation of serum tumor markers such as CA-125 can be of use in identifying suspected malignancy and has been found to be correlated with epithelial ovarian cancer as well as, in EOPPC patients, with extent and stage of disease [12]. It is a valuable tool to use in monitoring disease progression, response to treatment, and relapse, as shown in this patient.

(Neo-) adjuvant chemotherapy agents used in ovarian cancer can be applied in EOPPC due to molecular similarities. Response rates of $79 \%$ in EOPPC patients to first-line platinum-based chemotherapy are comparable $[13,14]$.

Due to the importance of optimal primary cytoreduction with residual masses $<1.0 \mathrm{~cm}$ [15], an open approach is deemed inevitable. Additional hyperthermic intraperitoneal chemotherapy (HIPEC) may be considered to further improve prognosis and response rates of residual disease. Complete cytoreductive surgery is achieved in $89 \%$ of cases and is therefore comparable to epithelial ovarian cancer patients [16].

Lesions are serous well-differentiated in $>90 \%$ of patients [10], as is our case.

No distinct risk factors for the development of EOPPC have been identified, excepting BRCA 1 and 2 germline mutations [17]. Menczer et al. reported similar frequency distributions of BRCA $1 / 2$ mutation for both ovarian and primary peritoneal carcinoma, further indicating a shared molecular pathogenesis [18]. Carrier status was associated with earlier onset and multifocal disease.

\section{Conclusions}

EOPPC may be misdiagnosed as epithelial ovarian cancer, but should be considered in patients with peritoneal carcinomatosis, normal-sized ovaries, and no identifiable primary lesion. Still, to further improve survival, optimal cytoreductive surgery is essential and should be a priority in disease management.

\section{Conflict of interest}

None declared.

\section{References:}

1. Goodman MT, Shvetsov YB: Incidence of ovarian, peritoneal, and fallopian tube carcinomas in the United States, 1995-2004. Cancer Epidemiol Biomarkers Prev, 2009; 18(1): 132-39

2. Swerdlow M: Mesothelioma of the pelvic peritoneum resembling papillary cystadenocarcinoma of the ovary. Am J Obstet Gynecol, 1959; 77(1) 197-200

3. Kannerstein M, Churg J: Peritoneal mesothelioma. Hum Pathol, 1977; 8(1): 83-94
4. Bloss JD, Liao SY, Buller RE et al: Extraovarian peritoneal serous papillary carcinoma: A case-control retrospective comparison to papillary adenocarcinoma of the ovary. Gynecol Oncol, 1993; 50(3): 347-51

5. Dubernard G, Morice P, Rey A et al: Lymph node spread in stage III or IV primary peritoneal serous papillary carcinoma. Gynecol Oncol, 2005; 97(1): 136-41

6. Eltabbakh GH, Mount SL: Lymphatic spread among women with primary peritoneal carcinoma. J Surg Oncol, 2002; 81(3): 126-31 
7. Halperin R, Zehavi S, Langer R et al: Primary peritoneal serous papillary carcinoma: A new epidemiologic trend? A matched-case comparison with ovarian serous papillary cancer. Int J Gynecol Cancer, 2001; 11(5): 403-8

8. Ordóñez NG: Pathologic characterization and differential diagnosis of malignant peritoneal mesothelioma. Recent Results Cancer Res, 2007; 169 123-36

9. Yun W, Bae J: Primary peritoneal serous carcinoma, an extremely rare malignancy: A case report and review of the literature. Oncol Lett, 2016; 11(6): 4063-65

10. Eltabbakh GH, Werness BA, Piver S, Blumenson LE: Prognostic factors in extraovarian primary peritoneal carcinoma. Gynecol Oncol, 1998; 71(2): 230-39

11. Rakheja R, Makis W, Hickeson M: Extraovarian primary peritoneal carcinoma: Staging with 18F-FDG PET/CT. Abdom Imaging, 2012; 37(2): 304-8

12. Killackey MA, Davis AR: Papillary serous carcinoma of the peritoneal surface: Matched-case comparison with papillary serous ovarian carcinoma. Gynecol Oncol, 1993; 51(2): 171-74
13. Roh SY, Hong SH, Ko Y et al: Clinical characteristics of primary peritoneal carcinoma. Cancer Res Treat, 2007; 39(2): 65-68

14. Fukuda T, Imai K, Yamauchi M et al: Primary peritoneal cancer: Study of 14 cases and comparison with epithelial ovarian cancer. Eur J Gynaecol Oncol, 2015; 36(1): 49-53

15. Bristow RE: Survival effect of maximal cytoreductive surgery for advanced ovarian carcinoma during the platinum era: A meta-analysis. J Clin Oncol, 2002; 20(5): 1248-59

16. Morice P, Dubernard G, Rey A et al: Results of interval debulking surgery compared with primary debulking surgery in advanced stage ovarian cancer. J Am Coll Surg, 2003; 197(6): 955-63

17. Bandera CA, Muto MG, Schorge JO et al: BRCA1 gene mutations in women with papillary serous carcinoma of the peritoneum. Obstet Gynecol, 1998; 92(4 Pt 1): 596-600

18. Menczer J, Chetrit A, Barda G et al: Frequency of BRCA mutations in primary peritoneal carcinoma in Israeli Jewish women. Gynecol Oncol, 2003; 88(1): 58-61 